\title{
ON THE GENERALIZED RAMANUJAN-NAGELL EQUATION $x^{2}-D=2^{n+2}$
}

\author{
LE MAOHUA
}

\begin{abstract}
Let $D$ be a positive integer which is odd. In this paper we prove that the equation $x^{2}-D=2^{n+2}$ has at most three positive integer solutions $(x, n)$ except when $D=2^{2 m}-3 \cdot 2^{m+1}+1$, where $m$ is a positive integer with $m \geq 3$.
\end{abstract}

\section{INTRODUCTION}

Let $\mathbf{Z}, \mathbf{N}, \mathbf{Q}$ be the sets of integers, positive integers and rational numbers respectively. Let $D \in \mathbf{N}$ be odd, and let $N(D)$ denote the number of solutions $(x, n)$ of the generalized Ramanujan-Nagell equation

$$
\chi^{2}-D=2^{n+2}, \quad \chi>0, n>0 .^{1}
$$

In [1], Beukers proved that $N(D) \leq 4$. Simultaneously, he showed that if $N(D)>3$, then $D$ must be among the following types:

(I) $D=2^{2 m}-3 \cdot 2^{m+1}+1, m \in \mathbf{N}, m \geq 3$.

(II) $D=\left(\left(2^{2 m+1}-17\right) / 3\right)^{2}-32, m \in \mathbf{N}, m \geq 3$.

(III) $D=2^{2 m_{2}}+2^{2 m_{1}}-2^{m_{2}+m_{1}+1}-2^{m_{2}+1}-2^{m_{1}+1}+1,{ }^{2} \quad m_{1}, m_{2} \in \mathbf{N}$, $m_{2}>m_{1}+1>2$.

Moreover, equation (1) has exactly four solutions $(x, n)=\left(2^{m}-3,1\right)$, $\left(2^{m}-1, m\right),\left(2^{m}+1, m+1\right)$, and $\left(3 \cdot 2^{m}-1,2 m+1\right)$ if $D$ is of type I. In this paper, we completely determine all $D$ for which $N(D)=4$.

Theorem. If $D$ is of type $\mathrm{I}$, then $N(D)=4$ otherwise $N(D) \leq 3$.

\section{Preliminaries}

Lemma 1 [3, Formula 1.76]. For any $m \in \mathbf{N}$ and any complex numbers $\alpha, \beta$, we have

$$
\alpha^{m}+\beta^{m}=\sum_{i=0}^{[m / 2]}(-1)^{i}\left[\begin{array}{c}
m \\
i
\end{array}\right](\alpha+\beta)^{m-2 i}(\alpha \beta)^{i},
$$

Received by the editors March 30, 1990 and, in revised form, September 18, 1990.

1980 Mathematics Subject Classification (1985 Revision). Primary 11D61.

'Throughout this paper, "solution" and "positive solution" are the abbreviations for "integer solution" and "positive integer solution" respectively.

${ }^{2}$ In [1] there is a misprint in this expression. 
where

are positive integers.

$$
\left[\begin{array}{c}
m \\
i
\end{array}\right]=\frac{(m-i-1) ! m}{(m-2 i) ! i !}, \quad i=0, \ldots,\left[\frac{m}{2}\right],
$$

Lemma 2 [2, Theorem 6.10.3]. Let $a / b, a^{\prime} / b^{\prime}, a^{\prime \prime} / b^{\prime \prime} \in \mathbf{Q}$ be positive with $a b^{\prime}-a^{\prime} b= \pm 1$. If $a^{\prime \prime} / b^{\prime \prime}$ lies in the interval $\left(a / b, a^{\prime} / b^{\prime}\right)$, then there exist positive integers $c, c^{\prime}$ such that

$$
a^{\prime \prime}=c a+c^{\prime} a^{\prime}, \quad b^{\prime \prime}=c b+c^{\prime} b^{\prime} .
$$

Lemma 3. If $(U, V)$ is a positive solution of the equation

$$
U^{2}-2 V^{2}=1
$$

with $2^{m+1} \mid V$ for some $m \in \mathbf{N}$, then $U+V \sqrt{2}=(3+2 \sqrt{2})^{2^{m t}}$ for some $t \in \mathbf{N}$. Proof. Since $3+2 \sqrt{2}$ is the fundamental solution of equation (2), we have $U+V \sqrt{2}=(3+2 \sqrt{2})^{\gamma}$ for some $\gamma \in \mathbf{N}$. Then

$$
V=\sum_{i=0}^{((r-1) / 2)}\left(\begin{array}{c}
\gamma \\
2 i+1
\end{array}\right) 3^{\gamma-2 i-1} 2^{3 i+1}
$$

If $2^{m+1} \mid V$, then from (3) we see that $2 \mid \gamma$. Further, if $2^{\lambda} \| \gamma$, since

$$
\left(\begin{array}{c}
\gamma \\
2 j+1
\end{array}\right) 2^{3 j+1}=\gamma\left(\begin{array}{c}
\gamma-1 \\
2 j
\end{array}\right) \frac{2^{3 j+1}}{2 j+1} \equiv 0\left(\bmod 2^{\lambda+2}\right) \text { for } j>0,
$$

we obtain $\lambda \geq m$ by (3). The lemma is proved.

Let $d \in \mathbf{N}$ be nonsquare, and let $k \in \mathbf{Z}$ with $\operatorname{gcd}(k, d)=1$.

Lemma 4 [2, Theorem 10.8.2]. If $|k|<\sqrt{d}$ and $(X, Y)$ is a positive solution of the equation

$$
X^{2}-d Y^{2}=k, \quad \operatorname{gcd}(X, Y)=1,
$$

then $X / Y$ is a convergent of $\sqrt{d}$.

It is a well-known fact that the simple continued fraction of $\sqrt{d}$ can be expressed as $\left[a_{0}, a_{1}, \ldots, a_{s}\right]$, where $a_{0}=(\sqrt{d}), a_{s}=2 a_{0}$, and $a_{i}<2 a_{0}$ for $i=0, \ldots, s-1$.

Lemma 5. For any nonnegative integer $j$, let $p_{j} / q_{j}$ and $\gamma_{j}$ denote the $j$ th convergent and complete quotient of $\sqrt{d}$ respectively. Further let

$$
k_{j}=(-1)^{j-1}\left(p_{j}^{2}-d q_{j}^{2}\right) \text { and } \Delta_{j}=(-1)^{j}\left(p_{j-1} p_{j}-d q_{j-1} q_{j}\right) .
$$

Then we have:

(i) $k_{j}>0, \Delta_{j}>0$, and

$$
a_{j+1}=\left[\frac{\Delta_{j}+\sqrt{d}}{k_{j}}\right] .
$$

(ii) $k_{j}=1$ if and only if $a_{j+1}=2 a_{0}$.

(iii) Let

$$
t= \begin{cases}s-1, & \text { if } 2 \mid s, \\ 2 s-1, & \text { if } 2 \nmid s .\end{cases}
$$


Then $p_{t}+q_{t} \sqrt{d}$ is the fundamental solution of the equation

$$
u^{2}-d v^{2}=1 \text {. }
$$

(iv) For any $m \in \mathbf{N}, k_{m s+i}=k_{i}(i=0, \ldots, s-1)$.

(v) If $1<k<\sqrt{d}, 2 d \not \equiv 0(\bmod k)$ and equation (4) has solutions $(X, Y)$, then it has at least two positive solutions such that

$$
X<p_{t}, \quad Y<q_{t} .
$$

Proof. Since $\frac{p_{0}}{q_{0}}<\cdots<\frac{p_{2 m}}{q_{2 m}}<\frac{p_{2 m+2}}{q_{2 m+2}}<\cdots<\sqrt{d}<\cdots<\frac{p_{2 m+1}}{q_{2 m+1}}<\frac{p_{2 m-1}}{q_{2 m-1}}<\cdots<\frac{p_{1}}{q_{1}}$ for any $m \in \mathbf{N}$, we get $k_{j}>0$ and $\Delta_{j}>0$. Since $p_{j-1} q_{j}-p_{j} q_{j-1}=(-1)^{j}$, we have

$$
\begin{gathered}
p_{j}=\Delta_{j} q_{j}+k_{j} q_{j-1}, \quad d q_{j}=\Delta_{j} p_{j}+k_{j} p_{j-1}, \\
d=\Delta_{j}^{2}+k_{j-1} k_{j} .
\end{gathered}
$$

So we obtain

$$
\gamma_{j+1}=-\frac{p_{j-1}-q_{j-1} \sqrt{d}}{p_{j}-q_{j} \sqrt{d}}=-\frac{\left(p_{j-1}-q_{j-1} \sqrt{d}\right)\left(p_{j}+q_{j} \sqrt{d}\right)}{\left(p_{j}-q_{j} \sqrt{d}\right)\left(p_{j}+q_{j} \sqrt{d}\right)}=\frac{\Delta_{j}+\sqrt{d}}{k_{j}} .
$$

Since $a_{j+1}=\left[\gamma_{j+1}\right],(5)$ is proved by $(10)$.

If $k_{j}=1$, then from (8) we get

$$
p_{j} / q_{j}=\Delta_{j}+q_{j-1} / q_{j}
$$

From

$$
\left[\frac{q_{j-1}}{q_{j}}\right]= \begin{cases}1, & \text { if } j=1 \text { and } q_{0}=q_{1}=1, \\ 0, & \text { otherwise }\end{cases}
$$

we obtain $\Delta_{j}=a_{0}$ by (11), and $a_{j+1}=2 a_{0}$ by (5). On the other hand, if $a_{j+1}=2 a_{0}$, since $\Delta_{j}<\sqrt{d}$ by (9), then we have $k_{j}=1$ by (5). Thus (ii) is proved.

By (ii), (iii) is clear. In addition, (iv) is Theorem 10.8.3 of [2].

Let $(X, Y)$ be a solution of equation (4). Since $k>1$ implies $X Y \neq 0$, then $(|X|,|Y|)$ is a positive solution of equation (4). By Lemma 4, $|X| /|Y|$ is a convergent of $\sqrt{d}$ since $k<\sqrt{d}$. Hence $|X| /|Y|=p_{2 r+1} / q_{2 r+1} \quad(\gamma \geq 0)$. Then there exists the integers $a, i$ such that $a \geq 0,2 \mid a s, 2 \nmid i$, and $0<i<t$, since $k>1$. By (iv), we have $k_{i}=k$. It follows that $\left(p_{i}, q_{i}\right)$ is a positive solution of equation (4) with (7). Let

$$
X^{\prime}=p_{i} p_{t}-d q_{i} q_{t}, \quad Y^{\prime}=p_{i} q_{t}-p_{t} q_{i} .
$$

Then $X^{\prime}, Y^{\prime}$ are coprime integers. From

$$
X^{\prime 2}-d Y^{\prime 2}=\left(p_{i}^{2}-d q_{i}^{2}\right)\left(p_{t}^{2}-d q_{t}^{2}\right)=k,
$$

we see that $\left(X^{\prime}, Y^{\prime}\right)$ is a solution of equation (4). Further, $\left(X^{\prime}, Y^{\prime}\right)$ is a positive solution by

$$
0<X^{\prime}-Y^{\prime} \sqrt{d}=\left(p_{i}+q_{i} \sqrt{d}\right)\left(p_{t}-q_{t} \sqrt{d}\right)=\frac{p_{i}+q_{i} \sqrt{d}}{p_{t}+q_{t} \sqrt{d}}<1 .
$$

By Lemma $4, X^{\prime} / Y^{\prime}$ is a convergent of $\sqrt{d}$. From

$$
X^{\prime}+Y^{\prime} \sqrt{d}=\left(p_{i}-q_{i} \sqrt{d}\right)\left(p_{t}+q_{t} \sqrt{d}\right)<p_{t}+q_{t} \sqrt{d},
$$


we get $X^{\prime}<p_{t}$ and $Y^{\prime}<q_{t}$. If $\left(X^{\prime}, Y^{\prime}\right)=\left(p_{i}, q_{i}\right)$, since $\operatorname{gcd}\left(p_{i}, q_{i}\right)=1$, then from (12) we get

$$
p_{t}-1=c_{1} q_{i}, \quad d q_{t}=c_{1} p_{i}, \quad p_{t}+1=c_{2} p_{i}, \quad q_{t}=c_{2} q_{i}, \quad c_{1}, c_{2} \in \mathbf{N} .
$$

Since $c_{1} p_{i}=c_{2} d q_{i}$, we have $c_{1}=c q_{i}, c_{2} d=c p_{i}$, where $c \in \mathbf{N}$. Hence $2 d=c_{2} d p_{i}-c_{1} d q_{i}=c\left(p_{i}^{2}-d q_{i}^{2}\right)=c k \equiv 0(\bmod k)$, a contradiction. Therefore $\left(X^{\prime}, Y^{\prime}\right) \neq\left(p_{i}, q_{i}\right),(\mathrm{v})$ is proved.

Let $I(d)=\left\{\left(d_{1}, d_{2}\right) \mid d_{1}, d_{2} \in \mathbf{N}, d_{1} d_{2}=d, \operatorname{gcd}\left(d_{1}, d_{2}\right)=1\right\}$, and let $I^{\prime}(d)=I(d) \backslash\{(1, d)\}$.

Lemma 6 [4]. There exists at most one pair $\left(d_{1}, d_{2}\right) \in I^{\prime}(d)$ which make the equation

$$
d_{1} u^{\prime 2}-d_{2} v^{\prime 2}=1
$$

has solutions $\left(u^{\prime}, v^{\prime}\right)$.

Lemma 7 [2, Theorems 11.4.1 and 11.4.2]. Let $\left(d_{1}, d_{2}\right) \in I(d)$. If $(X, Y)$ is a solution of the equation

$$
d_{1} X^{2}-d_{2} Y^{2}=k, \quad \operatorname{gcd}(X, Y)=1
$$

then there exists a unique integer $l$ such that

$$
l=d_{1} \alpha X-d_{2} \beta Y, \quad 0<l \leq|k|,
$$

where $\alpha, \beta \in \mathbf{Z}$ with $\beta X-\alpha Y=1$. Such $l$ is called the characteristic number of the solution $(X, Y)$, and it will be denoted by $\langle X, Y\rangle$. If $\langle X, Y\rangle=l$, then we have

$$
d_{1} X \equiv-l Y \quad(\bmod k), \quad l^{2} \equiv d \quad(\bmod k), \quad \operatorname{gcd}\left(k, 2 l, \frac{l^{2}-d}{k}\right)=1 .
$$

Lemma 8 [2, Theorem 11.4.2]. Let $\left(X_{1}, Y_{1}\right),\left(X_{2}, Y_{2}\right)$ be solutions of equation (14). Then $\left\langle X_{1}, Y_{1}\right\rangle=\left\langle X_{2}, Y_{2}\right\rangle$ if and only if

$$
X_{2} \sqrt{d_{1}}+Y_{2} \sqrt{d_{2}}=\left(X_{1} \sqrt{d_{1}}+Y_{1} \sqrt{d_{2}}\right)(u+v \sqrt{d})
$$

where $(u, v)$ is a solution of equation (6).

Lemma 9. If $2 \nmid d$ and the congruence

$$
l^{2} \equiv d \quad\left(\bmod 2^{m+2}\right), \quad 0<l \leq 2^{m+2}, \quad \operatorname{gcd}\left(2^{m+2}, 2 l, \frac{l^{2}-d}{2^{m+2}}\right)=1,
$$

has a solution $l$ for $m \in \mathbf{N}$, then it has exactly one solution $l^{\prime}=2^{m+2}-l$ with $l^{\prime} \neq l$.

Proof. Let $l^{\prime}$ be a solution of (15) with $l^{\prime} \neq l$. Since $2 \nmid d$ implies $2 \nmid$ $l l^{\prime}$, we get from $l^{2} \equiv l^{\prime 2} \equiv d\left(\bmod 2^{m+2}\right)$ that $l^{\prime} \equiv \delta l\left(\bmod 2^{m+1}\right)$, where $\delta \in\{-1,1\}$. If $\delta=1$, then $l^{\prime}=l+2^{m+1} t$ for some $t \in \mathbf{Z}$. Notice that $2 \nmid\left(l^{2}-d\right) / 2^{m+2}$ and $2 \nmid\left(l^{\prime 2}-d\right) / 2^{m+2}$. From

$$
\frac{l^{\prime 2}-d}{2^{m+2}}=\frac{l^{2}-d}{2^{m+2}}+l t+2^{m} t^{2}
$$


we get $2 \mid t$, and so $l^{\prime}=l$ since $0<l, l^{\prime} \leq 2^{m+2}$. This is a contradiction. Hence $\delta=-1$. Then $l^{\prime}=-l+2^{m+1} t$ for some $t \in \mathbf{Z}$. From

$$
\frac{l^{\prime 2}-d}{2^{m+2}}=\frac{l^{2}-d}{2^{m+2}}-l t+2^{m} t^{2}
$$

we obtain $l^{\prime}=2^{m+2}-l$ since $0<l, l^{\prime} \leq 2^{m+2}$. The lemma is proved.

Lemma 10. Let $m \in \mathbf{N}$, and let $\left(d_{1}, d_{2}\right) \in I(d)$. If $2 \nmid d$ and $\left(X_{0}, Y_{0}\right)$ is a solution of the equation

$$
d_{1} X^{2}-d_{2} Y^{2}=2^{m+2}, \quad \operatorname{gcd}(X, Y)=1,
$$

then all the solutions of equation (16) are given by

$$
X \sqrt{d_{1}}+Y \sqrt{d_{2}}=\left(X_{0} \sqrt{d_{1}}+Y_{0} \sqrt{d_{2}}\right)(u+v \sqrt{d}),
$$

where $(u, v)$ is an arbitrary solution of equation (6).

Proof. Under the assumption, $\left(X_{0},-Y_{0}\right)$ is a solution of equation (16) too. Let $l=\left\langle X_{0}, Y_{0}\right\rangle$. Then $\left\langle X_{0},-Y_{0}\right\rangle \equiv-l\left(\bmod 2^{m+2}\right)$. By Lemma 9 , we have either $\langle X, Y\rangle=\left\langle X_{0}, Y_{0}\right\rangle$ or $\langle X, Y\rangle=\left\langle X_{0},-Y_{0}\right\rangle$ for any solution $(X, Y)$ of equation (16). Thus, by Lemma 8 , the lemma is proved.

Lemma 11. If $2 \nmid d$ and the equation

$$
X^{2}-d Y^{2}=2^{z+2}, \quad \operatorname{gcd}(X, Y)=1, \quad Z>0,
$$

has solutions $(X, Y, Z)$, then it has a unique positive solution $\left(X_{1}, Y_{1}, Z_{1}\right)$ such that

$$
Z_{1} \leq Z, \quad 1<\frac{X_{1}+Y_{1} \sqrt{d}}{X_{1}-Y_{1} \sqrt{d}}<\left(u_{1}+v_{1} \sqrt{d}\right)^{2},
$$

where $Z$ runs over all solutions of equation $(17), u_{1}+v_{1} \sqrt{d}$ is the fundamental solution of equation (6). Such $\left(X_{1}, Y_{1}, Z_{1}\right)$ is called the least solution of equation (17). Moreover, all solutions of equation (17) are given by

$$
Z=Z_{1} t, \quad \frac{X+Y \sqrt{d}}{2}=\left(\frac{X_{1} \pm Y_{1} \sqrt{d}}{2}\right)^{t}(u+v \sqrt{d}),
$$

where $t$ is an arbitrary positive integer, $(u, v)$ is an arbitrary solution of equation (6).

Proof. Let $\left(X_{0}, Y_{0}, Z_{1}\right)$ be a solution of equation (17) with $Z_{1} \leq Z$. By Lemma 10 , all solutions of equation (17) with $Z=Z_{1}$ are given by

$$
X+Y \sqrt{d}=\left(X_{0} \pm Y_{0} \sqrt{d}\right)(u+v \sqrt{d}) .
$$

Since $u+v \sqrt{d}= \pm\left(u_{1}+v_{1} \sqrt{d}\right)^{\gamma} \quad(\gamma \in \mathbf{Z})$, we see from (19) that equation (17) has a unique positive solution $\left(X_{1}, Y_{1}, Z_{1}\right)$ which satisfy (18).

For any $t \in \mathbf{N}$, let

$$
\frac{X_{t}+Y_{t} \sqrt{d}}{2}=\left(\frac{X_{1}+Y_{1} \sqrt{d}}{2}\right)^{t}
$$


and let

$$
\varepsilon=\frac{X_{1}+Y_{1} \sqrt{d}}{2}, \quad \bar{\varepsilon}=\frac{X_{1}-Y_{1} \sqrt{d}}{2} .
$$

By Lemma 1, we have

$$
\begin{aligned}
& X_{t}=\varepsilon^{t}+\bar{\varepsilon}^{t}=\sum_{i=0}^{[t / 2]}(-1)^{i}\left[\begin{array}{l}
t \\
i
\end{array}\right](\varepsilon+\bar{\varepsilon})^{t-2 i}(\varepsilon \bar{\varepsilon})^{i}=\sum_{i=0}^{[t / 2]}(-1)^{i}\left[\begin{array}{l}
t \\
i
\end{array}\right] X_{1}^{t-2 i} 2^{z_{1} i} \\
& \left(\frac{\varepsilon-\bar{\varepsilon}}{\sqrt{d}} \sum_{i=0}^{(t-1) / 2}\left[\begin{array}{l}
t \\
i
\end{array}\right](\varepsilon-\bar{\varepsilon})^{t-2 i-1}(\varepsilon \bar{\varepsilon})^{i}\right. \\
& =Y_{1} \sum_{i=0}^{(t-1) / 2}\left[\begin{array}{l}
t \\
i
\end{array}\right]\left(d Y_{1}^{2}\right)^{(t-1) / 2-i} 2^{z_{1} i}, \quad \text { if } 2 \nmid t, \\
& Y_{t}=\frac{\varepsilon^{t}-\bar{\varepsilon}^{t}}{\sqrt{d}}=\left\{\frac{\varepsilon^{t^{\prime}}-\bar{\varepsilon}^{t^{\prime}}}{\sqrt{d}} \prod_{j=0}^{(\alpha-1)}\left(\varepsilon^{2^{j} t^{\prime}}+\bar{\varepsilon}^{2^{j} t^{\prime}}\right)=\left(Y_{1} \sum_{i=0}^{\left(t^{\prime}-1\right) / 2}\left[\begin{array}{l}
t^{\prime} \\
i
\end{array}\right]\left(d Y_{1}^{2}\right)^{\left(t^{\prime}-1\right) / 2-i} 2^{z_{1} i}\right)\right. \\
& \times \prod_{j=0}^{\alpha-1}\left(\sum_{i=0}^{\left[2^{j} t^{\prime} / 2\right]}(-1)^{i}\left(\begin{array}{c}
2^{j} t^{\prime} \\
i
\end{array}\right) X_{1}^{2^{j} t^{\prime}-2 i} 2^{z_{1} i}\right), \\
& \text { if } t=2^{\alpha} t^{\prime}, \alpha>0,2 \nmid t^{\prime} .
\end{aligned}
$$

Since $2 \nmid X_{1} Y_{1}$ implies $2 \nmid X_{t} Y_{t}$, we see that $\left(X_{t}, Y_{t}, Z_{1} t\right)$ is a solution of equation (17). Further, by Lemma 10, all solutions of equation (17) with $Z_{1} \mid Z$ are given by

$$
\begin{aligned}
Z=Z_{1} t, \quad \frac{X+Y \sqrt{d}}{2} & =\left(\frac{X_{t} \pm Y_{t} \sqrt{d}}{2}\right)(u+v \sqrt{d}) \\
& =\left(\frac{X_{1} \pm Y_{1} \sqrt{d}}{2}\right)^{t}(u+v \sqrt{d}) .
\end{aligned}
$$

Let $\left(X^{\prime}, Y^{\prime}, Z^{\prime}\right)$ be a solution of equation (17) with $Z_{1} \nmid Z^{\prime}$. Then $Z^{\prime}=$ $Z_{1} t+Z_{0}$, where $t, Z_{0} \in \mathbf{N}$ satisfy $Z_{0}<Z_{1}$. Let $l=\left\langle X_{t}, Y_{t}\right\rangle$, and let $l^{\prime}=$ $\left\langle X^{\prime}, Y^{\prime}\right\rangle$. By Lemma 7, we have

$$
\begin{aligned}
l^{2} \equiv d\left(\bmod 2^{z_{1} t+2}\right), & l^{\prime 2} \equiv d\left(\bmod 2^{z^{\prime}+2}\right), \\
X_{t} \equiv-l Y_{t}\left(\bmod 2^{z_{1} t+2}\right), & X^{\prime} \equiv-l^{\prime} Y^{\prime}\left(\bmod 2^{z^{\prime}+2}\right) .
\end{aligned}
$$

Since $2 \nmid l l^{\prime}$, we get $l^{\prime} \equiv \delta l\left(\bmod 2^{z_{1} t+2}\right)$, where $\delta \in\{-1,1\}$. From $(20)$,

$$
X_{t} X^{\prime}-\delta d Y_{t} Y^{\prime} \equiv 0 \quad\left(\bmod 2^{z_{1} t+2}\right), \quad X_{t} Y^{\prime}-\delta X^{\prime} Y_{t} \equiv 0 \quad\left(\bmod 2^{z_{1} t+2}\right) .
$$

There exists the integers $X^{\prime \prime}, Y^{\prime \prime}$ such that

$$
X_{t} X^{\prime}-\delta d Y_{t} Y^{\prime}=2^{z_{1} t+2} X^{\prime \prime}, \quad X_{t} Y^{\prime}-\delta X^{\prime} Y_{t}=2^{z_{1} t+2} Y^{\prime \prime} .
$$

Then

$$
X^{\prime} Y^{\prime}\left(X_{t}^{2}-d Y_{t}^{2}\right) \equiv 0 \quad\left(\bmod \operatorname{gcd}\left(2^{z_{1} t+2} X^{\prime \prime}, 2^{z_{1} t+2} Y^{\prime \prime}\right)\right)
$$

Since $2 \nmid X^{\prime} Y^{\prime}$, we get $2 \nmid \operatorname{gcd}\left(X^{\prime \prime}, Y^{\prime \prime}\right)$. From $(21)$ and

$$
2^{z^{\prime}+z_{1} t+4}=\left(X_{t}^{2}-d Y_{t}^{2}\right)\left(X^{\prime 2}-d Y^{\prime 2}\right)=\left(X_{t} X^{\prime}-\delta d Y_{t} Y^{\prime}\right)^{2}-d\left(X_{t} Y^{\prime}-\delta X^{\prime} Y_{t}\right)^{2}
$$


we have

$$
X^{\prime \prime 2}-d Y^{\prime \prime 2}=2^{z_{0}}
$$

Since $d \equiv 1(\bmod 8)$ implies $Z_{0}>2$, we see that $\left(X^{\prime \prime}, Y^{\prime \prime}, Z_{0}-2\right)$ is a solution of equation (17) with $Z<Z_{1}$, a contradiction. The lemma is proved.

Lemma 12. Let $\left(d_{1}, d_{2}\right) \in I^{\prime}(d)$. If $2 \nmid d$ and the equation

$$
d_{1} X^{\prime 2}-d_{2} Y^{\prime 2}=2^{z^{\prime}+2}, \quad \operatorname{gcd}\left(X^{\prime}, Y^{\prime}\right)=1, \quad Z^{\prime}>0,
$$

has solutions $\left(X^{\prime}, Y^{\prime}, Z^{\prime}\right)$ then equation (17) has solutions $(X, Y, Z)$. Moreover, if equation (13) has solutions $\left(u^{\prime}, v^{\prime}\right)$, then all solutions of equation (22) are given by

$$
Z^{\prime}=Z, \quad X^{\prime} \sqrt{d_{1}}+Y^{\prime} \sqrt{d_{2}}=(X+Y \sqrt{d})\left(u^{\prime} \sqrt{d_{1}}+v^{\prime} \sqrt{d_{2}}\right),
$$

where $(X, Y, Z)$ and $\left(u^{\prime}, v^{\prime}\right)$ are arbitrary solutions of equations (17) and (13) respectively. If equation (13) has no solution, then all solutions of equation (22) are given by

$$
Z^{\prime}=Z_{1}^{\prime} t^{\prime}, \quad \frac{X^{\prime} \sqrt{d_{1}}+Y^{\prime} \sqrt{d_{2}}}{2}=\left(\frac{X_{1}^{\prime} \sqrt{d_{1}} \pm Y_{1}^{\prime} \sqrt{d_{2}}}{2}\right)^{t^{\prime}}(u+v \sqrt{d})
$$

where $t^{\prime}$ is an arbitrary positive integer with $2 \nmid t^{\prime},(u, v)$ is an arbitrary solution of equation (6), $\left(X_{1}^{\prime}, Y_{1}^{\prime}, Z_{1}^{\prime}\right)$ is a unique positive solution of equation (22) such that

$$
Z_{1}^{\prime}=\frac{Z_{1}}{2}, \quad 1<\frac{X_{1}^{\prime} \sqrt{d_{1}}+Y_{1}^{\prime} \sqrt{d_{2}}}{X_{1}^{\prime} \sqrt{d_{1}}-Y_{1}^{\prime} \sqrt{d_{2}}}<\left(u_{1}+v_{1} \sqrt{d}\right)^{2},
$$

where $\left(X_{1}, Y_{1}, Z_{1}\right)$ is the least solution of equation (17), $u_{1}+v_{1} \sqrt{d}$ is the fundamental solution of equation (6). Such $\left(X_{1}^{\prime}, Y_{1}^{\prime}, Z_{1}^{\prime}\right)$ is called the least solution of equation (22).

Proof. Let $\left(X^{\prime}, Y^{\prime}, Z^{\prime}\right)$ be a solution of equation (22). Then

$$
\left(\frac{d_{1} X^{\prime 2}+d_{2} Y^{\prime 2}}{2}\right)^{2}-d\left(X^{\prime} Y^{\prime}\right)^{2}=2^{2 z^{\prime}+2}
$$

where $\left(d_{1} X^{\prime 2}+d_{2} Y^{\prime 2}\right) / 2$ and $X^{\prime} Y^{\prime}$ are coprime integers. It follows that equation (17) has solutions.

If equation (13) has solutions, then (23) gives out all solutions of equation (22) clearly.

If equation (13) has no solution, by Lemma 10, equation (22) has a unique positive solution $\left(X_{1}^{\prime}, Y_{1}^{\prime}, Z_{1}^{\prime}\right)$ satisfies $Z_{1}^{\prime} \leq Z^{\prime}$ and

$$
1<\frac{X_{1}^{\prime} \sqrt{d_{1}}+Y_{1}^{\prime} \sqrt{d_{2}}}{X_{1}^{\prime} \sqrt{d_{1}}-Y_{1}^{\prime} \sqrt{d_{2}}}<\left(u_{1}+v_{1} \sqrt{d}\right)^{2}
$$

where $Z^{\prime}$ runs over all solutions of equation (22). Since $\left(\left(d_{1} X_{1}^{\prime 2}+d_{2} Y_{1}^{\prime 2}\right) / 2\right.$, $X_{1}^{\prime} Y_{1}^{\prime}, 2 Z_{1}^{\prime}$ ) is a solution of equation (17), by Lemma 11 , we have $2 Z_{1}^{\prime}=Z_{1} t$ for some $t \in \mathbf{N}$. If $t>1$, then $Z_{1}^{\prime} \geq Z_{1}$. By such that same argument as in the proof of Lemma 11, there exists the integers $X^{\prime \prime}, Y^{\prime \prime}$ satisfy

$$
d_{1} X^{\prime \prime 2}-d_{2} Y^{\prime \prime 2}=2^{z_{1}^{\prime}-z_{1}}, \quad \operatorname{gcd}\left(X^{\prime \prime}, Y^{\prime \prime}\right)=1 \text {. }
$$


Recalling that $Z_{1}^{\prime} \leq Z_{1}$ and equation (13) has no solution. It is impossible. Therefore $t=1$ and (25) is proved.

Finally, by such the same argument as in the proof of Lemma 11 , we can prove that all solutions of equation (22) are given by (24). The proof is complete.

Lemma 13. If $2 \nmid d$, then there exists at most two distinct pairs $\left(d_{1}, d_{2}\right) \in I(d)$ which make equation (16) have solutions $(X, Y)$.

Proof. Let $\left(d_{1}, d_{2}\right),\left(d_{1}^{\prime}, d_{2}^{\prime}\right) \in I(d)$ with $\left(d_{1}, d_{2}\right) \neq\left(d_{1}^{\prime}, d_{2}^{\prime}\right)$. We assume that the equations

$$
d_{1} X^{2}-d_{2} Y^{2}=2^{m+2}, \quad \operatorname{gcd}(X, Y)=1,
$$

and

$$
d_{1}^{\prime} X^{\prime 2}-d_{2}^{\prime} Y^{\prime 2}=2^{m+2}, \quad \operatorname{gcd}\left(X^{\prime}, Y^{\prime}\right)=1,
$$

have solutions $(X, Y)$ and $\left(X^{\prime}, Y^{\prime}\right)$ respectively. Let $l=\langle X, Y\rangle$ and $l^{\prime}=$ $\left\langle X^{\prime}, Y^{\prime}\right\rangle$. By Lemma 9, we have $l^{\prime} \equiv \delta l\left(\bmod 2^{m+2}\right)$, where $\delta \in\{-1,1\}$. Further, by Lemma 7, we have

$$
d_{1} X \equiv-l Y \quad\left(\bmod 2^{m+2}\right), \quad d_{1}^{\prime} X^{\prime} \equiv-l^{\prime} Y^{\prime} \equiv-\delta l Y^{\prime} \quad\left(\bmod 2^{m+2}\right) .
$$

Hence

$$
\begin{gathered}
d_{1} d_{1}^{\prime} X X^{\prime} \equiv \delta l^{2} Y Y^{\prime} \equiv \delta d Y Y^{\prime}\left(\bmod 2^{m+2}\right), \\
d_{1} \delta l X Y^{\prime} \equiv d_{1}^{\prime} l X^{\prime} Y\left(\bmod 2^{m+2}\right) .
\end{gathered}
$$

Let $d_{11}=\operatorname{gcd}\left(d_{1}, d_{1}^{\prime}\right), d_{12}=\operatorname{gcd}\left(d_{1}, d_{2}^{\prime}\right), d_{21}=d_{1}^{\prime} / d_{11}, d_{22}=d_{2}^{\prime} / d_{12}$. Since $d_{1} d_{2}=d_{1}^{\prime} d_{2}^{\prime}=d$, then $d_{1}=d_{11} d_{12}, d_{2}=d_{21} d_{22}, d_{1}^{\prime}=d_{11} d_{21}, d_{2}^{\prime}=d_{12} d_{22}$. Notice that $2 \nmid d l l^{\prime}$. We obtain from (28) that

$$
d_{11} X X^{\prime}-\delta d_{22} Y Y^{\prime} \equiv d_{12} X Y^{\prime}-\delta d_{21} X^{\prime} Y \equiv 0 \quad\left(\bmod 2^{m+2}\right),
$$

whence we get

$$
d_{11} X X^{\prime}-\delta d_{22} Y Y^{\prime}=2^{m+2} X^{\prime \prime}, \quad d_{12} X Y^{\prime}-\delta d_{21} X^{\prime} Y=2^{m+2} Y^{\prime \prime},
$$

where $X^{\prime \prime}, Y^{\prime \prime} \in \mathbf{Z}$. By (26) and (27),

$$
\begin{aligned}
2^{2 m+4} & =\left(d_{1} X^{2}-d_{2} Y^{2}\right)\left(d_{1}^{\prime} X^{\prime 2}-d_{2}^{\prime} Y^{\prime 2}\right) \\
& =d_{1}^{\prime \prime}\left(d_{11} X X^{\prime}-\delta d_{22} Y Y^{\prime}\right)^{2}-d_{2}^{\prime \prime}\left(d_{12} X Y^{\prime}-\delta d_{21} X^{\prime} Y\right)^{2},
\end{aligned}
$$

where $d_{1}^{\prime \prime}=d_{12} d_{21}, d_{2}^{\prime \prime}=d_{11} d_{22}$ with $d_{1}^{\prime \prime} d_{2}^{\prime \prime}=d$. Substituting (29) into (30), we get

$$
d_{1}^{\prime \prime} X^{\prime \prime 2}-d_{2}^{\prime \prime} Y^{\prime \prime 2}=1
$$

Since $\left(d_{1}, d_{2}\right) \neq\left(d_{1}^{\prime}, d_{2}^{\prime}\right)$ implies $d_{12}>1, d_{1}^{\prime \prime}>1$, and $\left(d_{1}^{\prime \prime}, d_{2}^{\prime \prime}\right) \in I^{\prime}(d)$. From $(31)$, such $\left(d_{1}^{\prime \prime}, d_{2}^{\prime \prime}\right)$ is unique by Lemma 6 . We note that if $\left(d_{1}, d_{2}\right)$ is fixed, then the corresponding $\left(d_{1}^{\prime \prime}, d_{2}^{\prime \prime}\right)$ are different for some distinct $\left(d_{1}^{\prime}, d_{2}^{\prime}\right)$. This implies the lemma.

\section{FURTHER PRELIMINARY LEMMAS}

Throughout this section, we assume that $D$ is a nonsquare. Notice that the least solution of the equation

$$
X^{2}-D Y^{2}=2^{z+2}, \quad \operatorname{gcd}(X, Y)=1, \quad Z>0,
$$

is unique. By Lemmas 12 and 13, the following two lemmas are clear. 
Lemma 14. If there exists two distinct pairs $\left(D_{1}, D_{2}\right) \in I^{\prime}(D)$ which make the equation

$$
D_{1} X^{\prime 2}-D_{2} Y^{\prime 2}=2^{Z^{\prime}+2}, \quad \operatorname{gcd}\left(X^{\prime}, Y^{\prime}\right)=1, \quad Z^{\prime}>0
$$

have solutions $\left(X^{\prime}, Y^{\prime}, Z^{\prime}\right)$, then the least solution $\left(X_{1}, Y_{1}, Z_{1}\right)$ of equation (32) satisfies $2 \mid Z_{1}$.

Lemma 15. There exists at most three distinct pairs $\left(D_{1}, D_{2}\right) \in I^{\prime}(D)$ which make equation (33) have solutions $\left(X^{\prime}, Y^{\prime}, Z^{\prime}\right)$.

Lemma 16 [1, Lemma 7]. Suppose there exist integers $a, b, A, B, m$ such that

$$
\frac{A+B \sqrt{D}}{2}=\left(\frac{a+b \sqrt{D}}{2}\right)^{m}, \quad m>1, b \neq 0, a \equiv D b \quad(\bmod 2) .
$$

If $D>1$ and $D \equiv 1(\bmod 8)$, then $|B|>1$ except when $m=2$ and $a, b \in$ $\{-1,1\}$.

Lemma 17. If $(x, n)$ is a solution of equation $(1)$, then $(x, 1, n)$ is a solution of equation (32). Let $\left(X_{1}, Y_{1}, Z_{1}\right)$ be the least solution of equation (32), and let $u_{1}+v_{1} \sqrt{D}$ be the fundamental solution of the equation

$$
u^{2}-D v^{2}=1 \text {. }
$$

Further let

$$
\begin{array}{cc}
\varepsilon=\frac{X_{1}+Y_{1} \sqrt{D}}{2}, & \bar{\varepsilon}=\frac{X_{1}-Y_{1} \sqrt{D}}{2}, \\
\rho=u_{1}+v_{1} \sqrt{D}, & \bar{\rho}=u_{1}-v_{1} \sqrt{D} .
\end{array}
$$

Then

$$
n=Z_{1} t, \quad \frac{x+\delta \sqrt{D}}{2}=\varepsilon^{t} \bar{\rho}^{s}, \quad \delta \in\{-1,1\}
$$

where $s, t \in \mathbf{Z}$ satisfy

(37) $\quad s \geq 0, \quad t>0, \quad \operatorname{gcd}(s, t)= \begin{cases}2, & \text { if } 2|s, 2| t \text { and } x=\frac{D+1}{2}, \\ 1, & \text { otherwise. }\end{cases}$

Proof. By Lemma 11, (36) holds for some $s, t \in \mathbf{Z}$ with $s \geq 0$ and $t>0$. Moreover, by Lemma 16, $s$ and $t$ satisfy (37). The lemma is proved.

Lemma 18. Under the assumption of Lemma 17 , we have $\delta \equiv x Y_{1} / X_{1}(\bmod 4)$. Proof. Let

$$
\frac{X+Y \sqrt{D}}{2}=\varepsilon^{t}, \quad u-v \sqrt{D}=\bar{\rho}^{s} .
$$

By Lemma 1 , we have $X, Y \in \mathbf{Z}$ satisfy

$$
\begin{aligned}
X & =\varepsilon^{t}+\bar{\varepsilon}^{t}=\sum_{i=0}^{[t / 2]}(-1)^{i}\left[\begin{array}{l}
t \\
i
\end{array}\right](\varepsilon+\bar{\varepsilon})^{t-2 i}(\varepsilon \bar{\varepsilon})^{i}=\sum_{i=0}^{[t / 2]}(-1)^{i}\left[\begin{array}{l}
t \\
i
\end{array}\right] X_{1}^{t-2 i} 2^{Z_{1} i} \\
& \equiv \begin{cases}X_{1}^{t}-2 t X_{1}^{t-2}(\bmod 4), & \text { if } Z_{1}=1 \\
X_{1}^{t}(\bmod 4), & \text { if } Z_{1}>1,\end{cases}
\end{aligned}
$$


(40) $Y=\frac{\varepsilon^{t}-\bar{\varepsilon}^{t}}{\sqrt{D}} \equiv\left\{\begin{array}{l}Y_{1}^{t}+2 t Y_{1}^{t-2}(\bmod 4), \quad \text { if } Z_{1}=1,2 \nmid t, \\ \left(Y_{1}^{t^{\prime}}+2 t^{\prime} Y_{1}^{t^{\prime}-2}\right)\left(X_{1}^{t^{\prime}}-2 t^{\prime} X_{1}^{t^{\prime}-2}\right)(\bmod 4), \\ \quad \text { if } Z_{1}=1, t=2^{\alpha} t^{\prime}, \alpha>0,2 \nmid t^{\prime}, \\ Y_{1}^{t}(\bmod 4), \quad \text { if } Z_{1}>1,2 \nmid t, \\ Y_{1}^{t^{\prime}} X_{1}^{t-t^{\prime}}(\bmod 4) \quad \text { if } Z_{1}>1, t=2^{\alpha} t^{\prime}, \alpha>0,2 \nmid t^{\prime},\end{array}\right.$ since $D \equiv 1(\bmod 8)$. Notice that $4 \mid v$ when $D \equiv 1(\bmod 8)$. Then from

$$
\frac{x+\delta \sqrt{D}}{2}=\left(\frac{X+Y \sqrt{D}}{2}\right)(u-v \sqrt{D}),
$$

we get $x=X u-D Y v \equiv X u(\bmod 4)$ and $\delta=Y u-X v \equiv Y u(\bmod 4)$, and so

$$
\delta \equiv \frac{x Y}{X} \quad(\bmod 4)
$$

Since $X_{1}^{2} \equiv D Y_{1}^{2}(\bmod 8)$, substituting (39) and (40) into (42), the lemma is proved.

Lemma 19. If $(x, n)$ is a solution of equation (1) with $2 \mid n$, then $2^{n}<D^{2} / 16$. Proof. Under the assumption, we have $x+2^{n / 2+1}=D_{1}$ and $x-2^{n / 2+1}=D_{2}$, where $\left(D_{1}, D_{2}\right) \in I(D)$. It follows that $2^{n / 2+2}=D_{1}-D_{2} \leq D-1<D$. Thus the lemma.

Lemma 20. If $(x, n)$ is a solution of equation (1) with $2 \nmid n$, then $2 \nmid Z_{1} t$ and $\left(x, 2^{Z_{1}((t-1) / 2)}\right)$ is a solution of the equation

$$
x^{\prime 2}-2^{Z_{1}+2} y^{\prime 2}=D, \quad \operatorname{gcd}\left(x^{\prime}, y^{\prime}\right)=1,
$$

satisfying

$$
\left\langle x^{\prime}, 2^{Z_{1}((t-1) / 2)}\right\rangle \equiv \begin{cases}-X_{1}(\bmod D), & \text { if } 2 \mid s, \\ -X_{1} u_{1}(\bmod D), & \text { if } 2 \nmid s .\end{cases}
$$

Proof. By Lemma 7, we have

$$
\left\langle x, 2^{z_{1}((t-1) / 2)}\right\rangle \equiv-\frac{x}{2^{z_{1}((t-1) / 2)}} \quad(\bmod D) .
$$

From (38) and (41), we get

$$
\begin{aligned}
x & \equiv X u \equiv \frac{X_{1}^{t} u_{1}^{s}}{2^{t-1}} \equiv 2^{z_{1}((t-1) / 2)} X_{1} u_{1}^{s} \\
& \equiv \begin{cases}2^{z_{1}((t-1) / 2)} X_{1}(\bmod D), & \text { if } 2 \mid s, \\
2^{z_{1}((t-1) / 2)} X_{1} u_{1}(\bmod D), & \text { if } 2 \nmid s,\end{cases}
\end{aligned}
$$

since $2 \nmid Z_{1} t, X_{1}^{2} \equiv 2^{z_{1}+2}(\bmod D)$ and $u_{1}^{2} \equiv 1(\bmod D)$. Substituting (45) into (44), we obtain the lemma.

Lemma 21. Let $\left(X_{1}, Y_{1}, Z_{1}\right)$ be the least solution of equation (32). If $2^{r z_{1}+2}<$ $\sqrt{D}$ for some $r \in \mathbf{N}$, then the fundamental solution $\rho=u_{1}+v_{1} \sqrt{D}$ of equation (34) satisfies $\rho>D^{r / 2} / 2^{2 r-2}$.

Proof. By Lemma 11, there exists $X_{i}, Y_{i} \in \mathbf{Z}(i=1, \ldots, \gamma)$ such that

$$
X_{i}^{2}-D Y_{i}^{2}=2^{z_{1} i+2}, \quad \operatorname{gcd}\left(X_{i}, Y_{i}\right)=1, \quad i=1, \ldots, r .
$$


Since $2^{r z_{1}+2}<\sqrt{D}$, by $(\mathrm{v})$ of Lemma $5, \sqrt{D}$ has $2 r$ convergents $p_{s_{i}} / q_{s_{i}}$ and $p_{t_{i}} / q_{t_{i}}(i=1, \ldots, \gamma)$ such that

$$
k_{s_{i}}=k_{t_{i}}=2^{z_{1} i+2}, \quad 2 \nmid s_{i} t_{i}, 0<s_{i}, t_{i}<t, i=1, \ldots, \gamma,
$$

where $t$ was defined in (iii) of Lemma 5. Therefore, by (i) of Lemma 5, we have

$$
\begin{aligned}
& a_{s_{i}+1}=\left[\frac{\Delta_{s_{i}}+\sqrt{D}}{k_{s_{i}}}\right]>\frac{\sqrt{D}}{2^{z_{1} i+2}}, \\
& a_{t_{i}+1}=\left[\frac{\Delta_{t_{i}}+\sqrt{D}}{k_{t_{i}}}\right]>\frac{\sqrt{D}}{2^{z_{1} i+2}}, \quad i=1, \ldots, r .
\end{aligned}
$$

Notice where $p_{0}=a_{0}, p_{1}=a_{0} a_{1}+1$, and $p_{j+2}=a_{j+2} p_{j+1}+p_{j}$ for $j \geq 0$. By (iii) of Lemma 5 , we deduce from (46) that

$$
\begin{aligned}
\rho & >u_{1}=p_{t}>\prod_{j=0}^{t} a_{j} \geq a_{0} \prod_{i=1}^{\gamma} a_{s_{i}} a_{t_{i}} \\
& >a_{0}\left(\prod_{i=1}^{\gamma} \frac{\sqrt{D}}{2^{z_{1} i+2}}\right)^{2}=\frac{a_{0} D^{r}}{2^{r(r+1) z_{1}+4 r}}>\frac{D^{r / 2}}{2^{2 r-2}},
\end{aligned}
$$

since $a_{0}=[\sqrt{D}]$. The lemma is proved.

Lemma 22 [1, Lemma 6 and the proof of Theorem 3]. Let $(x, n),\left(x^{\prime}, n^{\prime}\right)$, $\left(x^{\prime \prime}, n^{\prime \prime}\right)$ be three solutions of equation (1) with $n^{\prime \prime}>n^{\prime}>n$. We have:

(i) If $x^{\prime}-x=2$, then either $D$ is of type I or $D$ is of type III and $\left(x, x^{\prime}\right)=$ $\left(2^{m_{2}}-2^{m_{1}}-1,2^{m_{2}}-2^{m_{1}}+1\right)$.

(ii) If $x^{\prime}-x=4$, then $D$ is of type I.

(iii) If $D$ is of type II and $\left(x, x^{\prime}, x^{\prime \prime}\right)=\left(\left(2^{2 m+1}-17\right) / 3,\left(2^{2 m+1}+1\right) / 3\right.$, $\left.\left(17 \cdot 2^{2 m+1}-1\right) / 3\right)$, then $n^{\prime \prime}=2 n^{\prime}+3$.

(iv) With the exception of above cases, $x^{\prime}-x \geq 6$ and $n^{\prime \prime} \geq 2 n^{\prime}+53$.

Lemma 23 [1, Corollaries 1 and 2]. If $(\chi, n)$ is a solution of equation (1), then $n<433+(10 \log D) / \log 2$. Moreover, if $D<2^{96}$, then $n<16+$ $(2 \log D) / \log 2$.

\section{PROOF OF THEOREM}

By Theorems 3 and 4 of [1], it suffices to prove that $N(D)=3$ while $D \geq$ $10^{12}$ and $D$ is of types II or III. Moreover, if $D$ is a square, then $N(D) \leq 1$. We may assume that $D$ is not a square.

Assertion 1. If $D$ is of type II, then $N(D)=3$.

Proof. In this case, equation (1) has three solutions

$$
\begin{aligned}
& \left(x_{1}, n_{1}\right)=\left(\frac{2^{2 m+1}-17}{3}, 3\right), \quad\left(x_{2}, n_{2}\right)=\left(\frac{2^{2 m+1}}{3}, 2 m+1\right), \\
& \left(x_{3}, n_{3}\right)=\left(\frac{17 \cdot 2^{2 m+1}-1}{3}, 4 m+5\right)
\end{aligned}
$$


By the proof of Theorem 3 of [1], if $N(D)>3$, then equation (1) has another solution $\left(x_{4}, n_{4}\right)$ with $n_{4}>n_{3}$. By Lemmas 19 and 22 , we see that $2 \nmid n_{4}$. Let $\left(X_{1}, Y_{1}, Z_{1}\right)$ be the least solution of (32), and let $\varepsilon, \bar{\varepsilon}, \rho, \bar{\rho}$ be defined as in (35). Then, by Lemma 17, we have

$$
n_{i}=Z_{i} t_{i}, \quad \frac{\chi_{i}+\delta_{i} \sqrt{D}}{2}=\varepsilon^{t_{i}} \bar{\rho}^{s_{i}}, \quad \delta_{i} \in\{-1,1\}, i=1, \ldots, 4,
$$

where $s_{i}, t_{i} \in \mathbf{Z}(i=1, \ldots, 4)$ satisfy

$$
s_{i} \geq 0, \quad t_{i}>0, \quad \operatorname{gcd}\left(s_{i}, t_{i}\right)=1, \quad i=1, \ldots, 4 .
$$

We see from (47) and (48) that equation (43) has three solutions $\left(x_{j}, 2^{z_{1}\left(\left(t_{j}-1\right) / 2\right)}\right)$ $(j=2,3,4)$. Let $l_{j}=\left\langle x_{j}, 2^{z_{1}\left(\left(t_{j}-1\right) / 2\right)}\right\rangle \quad(j=2,3,4)$. By Lemma 7 , we get from (47) and (48) that

$$
\begin{aligned}
l_{2}-l_{3} & \equiv-\frac{2^{2 m+1}+1}{3 \cdot 2^{z_{1}\left(\left(t_{2}-1\right) / 2\right)}}+\frac{17 \cdot 2^{2 m+1}-1}{3 \cdot 2^{z_{1}\left(\left(t_{3}-1\right) / 2\right)}} \\
& \equiv-\frac{2^{\left(z_{1}-1\right) / 2}}{3 \cdot 2^{2 m+2}}\left(2^{3 m+3}-17 \cdot 2^{2 m+1}+2^{m+2}+1\right) \not \equiv 0(\bmod D) .
\end{aligned}
$$

It follows that $l_{2} \neq l_{3}$. Further, by Lemma 20, we have either $l_{4}=l_{2}$ or $l_{4}=l_{3}$. Furthermore, by Lemma 8, we get

$$
\begin{aligned}
x_{4}+ & 2^{z_{1}\left(\left(t_{4}-1\right) / 2\right) \sqrt{2^{z_{1}+2}}} \\
& = \begin{cases}\left(x_{2}+2^{z_{1}\left(\left(t_{2}-1\right) / 2\right)} \sqrt{2^{z_{1}+2}}\left(U^{\prime}+V^{\prime} \sqrt{2^{z_{1}+2}}\right),\right. & \text { if } l_{4}=l_{2}, \\
\left(x_{3}+2^{z_{1}\left(\left(t_{3}-1\right) / 2\right)} \sqrt{2^{z_{1}+2}}\right)\left(U^{\prime}+V^{\prime} \sqrt{2^{z_{1}+2}}\right), & \text { if } l_{4}=l_{3},\end{cases}
\end{aligned}
$$

and hence

$$
2^{z_{1}\left(\left(t_{4}-1\right) / 2\right)}= \begin{cases}x_{2} V^{\prime}+2^{z_{1}\left(\left(t_{2}-1\right) / 2\right)} U^{\prime}, & \text { if } l_{4}=l_{2}, \\ x_{3} V^{\prime}+2^{z_{1}\left(\left(t_{3}-1\right) / 2\right)} U^{\prime}, & \text { if } l_{4}=l_{3},\end{cases}
$$

where $\left(U^{\prime}, V^{\prime}\right)$ is a positive solution of the equation

$$
U^{\prime 2}-2^{z_{1}+2} V^{\prime 2}=1 \text {. }
$$

Since $t_{3}>t_{2}$, we obtain

$$
2^{z_{1}\left(\left(t_{2}-1\right) / 2\right)} \mid V^{\prime}
$$

by (50). On applying Lemma 3 with (52), we have

$$
U^{\prime}+V^{\prime} \sqrt{2^{z_{1}+2}}=(3+2 \sqrt{2})^{2^{m_{\gamma}}}, \quad \gamma \in \mathbf{N},
$$

since $Z_{1} t_{2}=2 m+1$. From (53), we deduce $2 U^{\prime}>2^{5 \cdot 2^{m-1}}$ and

$$
n_{4}>2 m+1+5 \cdot 2^{m}
$$

by (47), (48), and (50). On the other hand, by Lemma 23 , we have

$$
n_{4}<433+10 \frac{\log D}{\log 2}<433+40 m
$$

since $D<2^{4 m}$. The combination of (54) and (55) yields $m \leq 7$ and $D<$ $2^{4 m} \leq 2^{28}<10^{12}$. Thus the assertion is proved. 
Assertion 2. If $D$ is of type III, then $N(D)=3$.

Proof. In this case, equation (1) has three solutions

$$
\begin{aligned}
& \left(x_{1}, n_{1}\right)=\left(2^{m_{2}}-2^{m_{1}}-1, m_{1}\right), \\
& \left(x_{2}, n_{2}\right)=\left(2^{m_{2}}-2^{m_{1}}+1, m_{2}\right), \\
& \left(x_{3}, n_{3}\right)=\left(2^{m_{2}}+2^{m_{1}}-1, m_{2}+m_{1}\right) .
\end{aligned}
$$

If $N(D)>3$, then equation (1) has another solution $\left(\chi_{4}, n_{4}\right)$ with $n_{4}>n_{3}$. Moreover, then (48) and (49) still hold by Lemma 17.

When $2 \mid m_{1}$ and $2 \mid m_{2}$, we get from (56) that

$$
D_{11}-D_{12}=2^{m_{1} / 2+2}, \quad D_{21}-D_{22}=2^{m_{2} / 2+2},
$$

where

$$
\begin{array}{ll}
D_{11}=2^{m_{2}}-2^{m_{1}}+2^{m_{1} / 2+1}-1, & D_{12}=2^{m_{2}}-2^{m_{1}}-2^{m_{1} / 2+1}-1, \\
D_{21}=2^{m_{2}}+2^{m_{2} / 2+1}-2^{m_{1}}+1, & D_{22}=2^{m_{2}}-2^{m_{2} / 2+1}-2^{m_{1}}+1 .
\end{array}
$$

Since $\left(D_{11}, D_{12}\right),\left(D_{21}, D_{22}\right) \in I^{\prime}(D)$ and $\left(D_{11}, D_{12}\right) \neq\left(D_{21}, D_{22}\right)$, by Lemma 14 , the least solution of equation (32) satisfies $2 \mid Z_{1}$. Therefore, $2 \mid n_{4}$ by (48). Then we have

$$
D_{31}-D_{32}=2^{\left(m_{2}+m_{1}\right) / 2+2}, \quad D_{41}-D_{42}=2^{n_{4} / 2+2},
$$

where

$$
\begin{array}{ll}
D_{31}=2^{m_{2}}+2^{\left(m_{2}+m_{1}\right) / 2+1}+2^{m_{1}}-1, & D_{32}=2^{m_{2}}-2^{\left(m_{2}+m_{1}\right) / 2+1}+2^{m_{1}}-1, \\
D_{41}=x_{4}+2^{n_{4} / 2+1}, & D_{42}=x_{4}-2^{n_{4} / 2+1} .
\end{array}
$$

Since $\left(D_{31}, D_{32}\right),\left(D_{41}, D_{42}\right) \in I^{\prime}(D)$, and $\left(D_{i 1}, D_{i 2}\right)(i=1, \ldots, 4)$ are different, this implies that there exist four distinct pairs $\left(D_{1}, D_{2}\right) \in I^{\prime}(D)$ which make equation (33) have solutions. By Lemma 15, it is impossible.

When $2 \mid m_{1}$ and $2 \nmid m_{2}$, we have $2 \nmid Z_{1}$ by (48). If $2 \mid n_{4}$, since $2 \mid m_{1}$, we see from Lemma 14 that $2 \mid Z_{1}$, a contradiction. Therefore $2 \nmid n_{4}$ and equation (43) has three solutions $\left(x_{j}, 2^{z_{1}\left(\left(t_{j}-1\right) / 2\right)}\right) \quad(j=2,3,4)$. Let $l_{j}=\left\langle x_{j}, 2^{z_{1}\left(\left(t_{j}-1\right) / 2\right)}\right\rangle(j=2,3,4)$. From (56), we get

$$
\begin{aligned}
l_{2}-l_{3} & \equiv-\frac{2^{m_{2}}-2^{m_{1}}+1}{2^{z_{1}\left(\left(t_{2}-1\right) / 2\right)}}+\frac{2^{m_{2}}+2^{m-1}-1}{2^{z_{1}\left(\left(t_{3}-1\right) / 2\right)}} \\
& \equiv \frac{2^{\left(z_{1}-1\right) / 2}}{2^{\left(m_{2}+m_{1}-1\right) / 2}}\left(-2^{m_{1} / 2}\left(2^{m_{2}}-2^{m_{1}}+1\right)+\left(2^{m_{2}}+2^{m_{1}}-1\right)\right) \not \equiv 0(\bmod D) .
\end{aligned}
$$

It follows that $l_{2} \neq l_{3}$ and either $l_{4}=l_{2}$ or $l_{4}=l_{3}$ by Lemma 20. By such the same argument as in the proof of Assertion 1, then (50) and (52) still hold. Hence

$$
U^{\prime}+V^{\prime} \sqrt{2^{z_{1}+2}}=(3+2 \sqrt{2})^{2^{\left(m_{2}-1\right) / 2} \gamma}, \quad \gamma \in \mathbf{N},
$$

whence we get $2 U^{\prime}>2^{5 \cdot 2^{\left(m_{2}-3\right) / 2}}$. On applying this with $(50)$, we obtain

$$
n_{4}>m_{2}+5 \cdot 2^{\left(m_{2}-3\right) / 2} \text {. }
$$

On the other hand, since $\sqrt{D}<2^{m_{2}}$, we have

$$
n_{4}<433+10 \frac{\log D}{\log 2}<433+20 m_{2}
$$


by Lemma 23. The combination of (57) and (58) yields $m_{2} \leq 17$ and $D<$ $2^{34}<10^{12}$, which is in contradiction with the assumption.

Let $2 \nmid m_{1} m_{2}$ and $3.6 m_{1} \geq m_{2}$. Since $2 \mid m_{2}+m_{1}$, we have $2 \nmid n_{4}$, and equation (43) has three solutions $\left(x_{j}, 2^{z_{1}\left(\left(t_{j}-1\right) / 2\right)}\right)(j=1,2, \ldots, 4)$. Let $l_{j}=$ $\left\langle x_{j}, 2^{\left.z_{1}\left(t_{j}-1\right) / 2\right)}\right\rangle(j=1,2,4)$. By Lemma 7 , we obtain $l_{1} \neq l_{2}$. Furthermore, by Lemma 20, we have either $l_{4}=l_{1}$ or $l_{4}=l_{2}$. By such the same argument as in the case that $2 \mid m_{1}$ and $2 \nmid m_{2}$, we can prove $l_{4} \neq l_{2}$. If $l_{4}=l_{1}$, we have $x_{4}+2^{z_{1}\left(\left(t_{4}-1\right) / 2\right)} \sqrt{2^{z_{1}+2}}=\left(2^{m_{2}}-2^{m_{1}}-1+2^{z_{1}\left(\left(t_{1}-1\right) / 2\right)} \sqrt{2^{z_{1}+2}}\right)\left(U^{\prime}+V^{\prime} \sqrt{2^{z_{1}+2}}\right)$, whence we get

$$
2^{z_{1}\left(\left(t_{4}-1\right) / 2\right)}=\left(2^{m_{2}}-2^{m_{1}}-1\right) V^{\prime}+2^{z_{1}\left(\left(t_{1}-1\right) / 2\right)} U^{\prime},
$$

where $U^{\prime}, V^{\prime} \in \mathbf{N}$ satisfy $(51)$. Hence $2^{z_{1}\left(\left(t_{1}-1\right) / 2\right)} \mid V^{\prime}$ and

$$
2^{z_{1}\left(\left(t_{4}-t_{1}\right) / 2\right)}=\left(2^{m_{2}}-2^{m_{1}}-1\right) \frac{V^{\prime}}{2^{z_{1}\left(\left(t_{1}-1\right) / 2\right)}}+U^{\prime}
$$

Further, by Lemma 3, we have

$$
U^{\prime}+V^{\prime} \sqrt{2^{z_{1}+2}}=(3+2 \sqrt{2})^{2^{\left(m_{1}-1\right) / 2} \gamma}, \quad \gamma \in \mathbf{N},
$$

since $m_{1}=Z_{1} t_{1}$ and $2 \nmid Z_{1}$. Furthermore, we see from (60) that $U^{\prime} \equiv 1$ $(\bmod 8)$ and

$$
\frac{V^{\prime}}{2^{z_{1}\left(\left(t_{1}-1\right) / 2\right)}} \equiv 3^{2^{\left(m_{1}-1\right) / 2} r-1} \gamma \equiv 3 r \quad(\bmod 8)
$$

since $m_{1} \geq 3$. Hence, we obtain $\gamma \equiv 3(\bmod 8)$ by (59). It implies that $\gamma \geq 3$ and

$$
2 U^{\prime}>2^{15 \cdot 2^{\left(m_{1}-3\right) / 2}}
$$

by (60). On applying this with (59), we get

$$
n_{4}>m_{1}+15 \cdot 2^{\left(m_{1}-1\right) / 2}-2 .
$$

On the other hand, by Lemma 23 ,

$$
n_{4}<433+10 \frac{\log D}{\log 2}<433+20 m_{2} \leq 433+72 m_{1} .
$$

The combination of (61) and (62) yields $m_{1} \leq 13$ and $D<2^{2 m_{2}} \leq 2^{7.2 m_{1}}<2^{96}$. On applying Lemma 23 again, we have

$$
n_{4}<16+2 \frac{\log D}{\log 2}<16+4 m_{2} \leq 16+14.4 m_{1}
$$

On combining this with (61), we get $m_{1} \leq 5$ and $D<2^{36}<10^{12}$. Thus $N(D)=3$.

Using the same method, we can prove the assertion for the case that $2 \nmid m_{1}$, $2 \mid m_{2}$, and $m_{2} \leq 3.6 m_{1}$.

Let $2 \nmid m_{1}$ and $m_{2}>3.6 m_{1}$. We obtain from (48) that

$$
\left(\frac{x_{2}+\delta_{2} \sqrt{D}}{2}\right)^{t_{3}} \rho^{s_{2} t_{3}}=\left(\frac{x_{3}+\delta_{3} \sqrt{D}}{2}\right)^{t_{2}} \rho^{s_{3} t_{2}}
$$

Since $x_{2} \equiv 1(\bmod 4)$ and $x_{3} \equiv-1(\bmod 4)$, we have

$$
\delta_{2}=-\delta_{3}
$$


by Lemma 18. Since $2^{m_{2}}-2^{m_{1}}-2<\sqrt{D}<2^{m_{2}}-2^{m_{1}}-1$, we have

$$
t_{3} \log \frac{x_{2}+\sqrt{D}}{2}+t_{2} \log \frac{x_{3}+\sqrt{D}}{2}>t_{2} t_{3} \log 2^{z_{1}}
$$

by (48) and (56). Hence, from (63) and (64),

$$
\begin{aligned}
\left|s_{2} t_{3}-s_{3} t_{2}\right| \log \rho= & \left|t_{3} \log \frac{x_{2}+\delta_{2} \sqrt{D}}{2}-t_{2} \log \frac{x_{3}+\delta_{3} \sqrt{D}}{2}\right| \\
= & t_{3} \log \frac{x_{2}+\sqrt{D}}{2}+t_{2} \log \frac{x_{3}+\sqrt{D}}{2}-t_{2} t_{3} \log 2^{z_{1}} \\
< & t_{3} \log \frac{1}{2}\left(\left(2^{m_{2}}-2^{m_{1}}+1\right)+\left(2^{m_{2}}-2^{m_{1}}-1\right)\right) \\
& +t_{2} \log \frac{1}{2}\left(\left(2^{m_{2}}+2^{m_{1}}-1\right)+\left(2^{m_{2}}-2^{m_{1}}-1\right)\right)-t_{3} \log 2^{m_{2}} \\
< & t_{2} \log 2^{m_{2}} .
\end{aligned}
$$

Notice that only one of $n_{2}$ and $n_{3}$ is even. We see from (49) that $2 \nmid s_{2} t_{3}-s_{3} t_{2}$. If $\left|s_{2} t_{3}-s_{3} t_{2}\right|>1$, then $\left|s_{2} t_{3}-s_{3} t_{2}\right| \geq 3$ and

$$
3 \log \rho<t_{2} \log 2^{m_{2}}
$$

by (65). Recalling that $m_{2}=Z_{1} t_{2}$ and $2 \nmid Z_{1}$. Since $2^{m_{2}-1}<\sqrt{D}<2^{m_{2}}$, we get

$$
\sqrt{D}> \begin{cases}2^{\left(t_{2}-3\right) z_{1}+2}, & \text { if } Z_{1}=1 \\ 2^{\left(t_{2}-1\right) z_{1}+2}, & \text { if } Z_{1}>1 .\end{cases}
$$

By Lemma 21, we have

$$
\log \rho> \begin{cases}\left(t_{2}-3\right) \log \sqrt{D}-\left(t_{2}-4\right) \log 4, & \text { if } Z_{1}=1, \\ \left(t_{2}-1\right) \log \sqrt{D}-\left(t_{2}-2\right) \log 4, & \text { if } Z_{1}>1 .\end{cases}
$$

Recalling that $D \geq 10^{12}$. The combination of (66) and (67) yields

$$
t_{2} \leq \begin{cases}4, & \text { if } Z_{1}=1, \\ 2, & \text { if } Z_{1}>1,\end{cases}
$$

a contradiction. Thus

$$
s_{2} t_{3}-s_{3} t_{2}= \pm 1
$$

Let $\alpha=(\log (\varepsilon / \bar{\varepsilon})) / \log \rho^{2}$, and let

$$
\Lambda(x, n)=\log \frac{x+\sqrt{D}}{x-\sqrt{D}},
$$

for any solution $(x, n)$ of equation (1). Then we have

$$
\alpha-\frac{s_{i}}{t_{i}}=\frac{\delta_{i} \Lambda\left(x_{i}, n_{i}\right)}{t_{i} \log \rho^{2}}, \quad i=1, \ldots, 4,
$$

by (48). We see from (64) that $\alpha$ lies in the interval $\left(s_{2} / t_{2}, s_{3} / t_{3}\right)$. Moreover, since $t_{4}>t_{j}$ and $\Lambda\left(x_{4}, n_{4}\right)<\Lambda\left(x_{j}, n_{j}\right)$ for $j=2,3$, we see from (69) that $s_{4} / t_{4}$ lies in the interval $\left(s_{2} / t_{2}, s_{3} / t_{3}\right)$ too. By Lemma 2 , we get from (68) that

$$
t_{4}=c t_{2}+c^{\prime} t_{3}, \quad s_{4}=c s_{2}+c^{\prime} s_{3}, \quad c, c^{\prime} \in \mathbf{N} .
$$


From (48) and (70), we have

$$
\frac{x_{4}+\delta_{4} \sqrt{D}}{2}=\varepsilon^{t_{4}} \bar{\rho}^{s_{4}}=\left(\frac{x_{2}+\delta_{2} \sqrt{D}}{2}\right)^{c}\left(\frac{x_{3}+\delta_{3} \sqrt{D}}{2}\right)^{c^{\prime}}
$$

Let

$$
\frac{X_{2}+Y_{2} \sqrt{D}}{2}=\left(\frac{x_{2}+\delta_{2} \sqrt{D}}{2}\right)^{c}, \quad \frac{X_{3}+Y_{3} \sqrt{D}}{2}=\left(\frac{x_{3}+\delta_{3} \sqrt{D}}{2}\right)^{c^{\prime}}
$$

Then $X_{2}, Y_{2}, X_{3}, Y_{3}$ are integers. Let $\varepsilon_{2}=\left(x_{2}+\delta_{2} \sqrt{D}\right) / 2$, and $\bar{\varepsilon}_{2}=$ $\left(x_{2}-\delta_{2} \sqrt{D}\right) / 2$. Since $\varepsilon_{2}+\bar{\varepsilon}_{2}=x_{2} \equiv 1-2^{m_{1}}\left(\bmod 2^{m_{2}}\right)$ and $\varepsilon_{2} \bar{\varepsilon}_{2}=2^{m_{2}} \equiv 0$ $\left(\bmod 2^{m_{2}}\right)$, by Lemma 1 , we have

$$
\varepsilon_{2}^{m}+\bar{\varepsilon}_{2}^{m}=\sum_{i=0}^{[m / 2]}(-1)^{i}\left[\begin{array}{c}
m \\
i
\end{array}\right]\left(\varepsilon_{2}+\bar{\varepsilon}_{2}\right)^{m-2 i}\left(\varepsilon_{2} \bar{\varepsilon}_{2}\right)^{i} \equiv\left(1-2^{m_{1}}\right)^{m} \quad\left(\bmod 2^{m_{2}}\right)
$$

for any $m \in \mathbf{N}$. It follows that $X_{2} \equiv\left(1-2^{m_{1}}\right)^{c}\left(\bmod 2^{m_{2}}\right)$. Simultaneously, we have

$$
\begin{aligned}
Y_{2} & =\frac{\varepsilon_{2}^{c}-\varepsilon_{2}^{c}}{\sqrt{D}}=\delta_{2} \frac{\varepsilon_{2}^{c}-\bar{\varepsilon}_{2}^{c}}{\varepsilon_{2}-\bar{\varepsilon}_{2}} \\
& =\delta_{2}\left(\left(\varepsilon_{2}^{c-1}+\bar{\varepsilon}_{2}^{c-1}\right)+\varepsilon_{2} \bar{\varepsilon}_{2}\left(\frac{\varepsilon_{2}^{c-2}-\bar{\varepsilon}_{2}^{c-2}}{\varepsilon_{2}-\bar{\varepsilon}_{2}}\right)\right) \\
& \equiv \delta_{2}\left(\varepsilon_{2}^{c-1}+\bar{\varepsilon}_{2}^{c-1}\right) \equiv \delta_{2}\left(1-2^{m_{1}}\right)^{c-1} \quad\left(\bmod 2^{m_{2}}\right) .
\end{aligned}
$$

By the same argument, we can get $X_{3} \equiv\left(-1+2^{m_{1}}\right)^{c^{\prime}}\left(\bmod 2^{m_{2}}\right)$ and $Y_{3} \equiv$ $\delta_{3}\left(-1+2^{m_{1}}\right)^{c^{\prime}-1}\left(\bmod 2^{m_{2}}\right)$, since $x_{3}=2^{m_{2}}+2^{m_{1}}-1$. From $(64),(71)$ and (72),

$$
\begin{aligned}
2 \delta_{4} & =X_{2} Y_{3}+X_{3} Y_{2} \\
& \equiv \delta_{3}\left(1-2^{m_{1}}\right)^{c}\left(-1+2^{m_{1}}\right)^{c^{\prime}-1}+\delta_{2}\left(1-2^{m_{1}}\right)^{c-1}\left(-1+2^{m_{1}}\right)^{c^{\prime}} \\
& \equiv(-1)^{c^{\prime}} 2 \delta_{2}\left(1-2^{m_{1}}\right)^{c+c^{\prime}-1} \quad\left(\bmod 2^{m_{2}}\right) .
\end{aligned}
$$

It follows that

$$
\pm 1 \equiv\left(1-2^{m_{1}}\right)^{c+c^{\prime}-1} \quad\left(\bmod 2^{m_{2}-1}\right)
$$

whence we deduce that $c+c^{\prime}-1 \equiv 0\left(\bmod 2^{m_{2}-m_{1}-1}\right)$. Since $m_{1} \geq 3$ and $m_{2}>3.6 m_{1}$, we have $c+c^{\prime}-1>2^{2.6 m_{1}-1}>2^{6.8}>96$. Hence, from (48), (56) and (70), we get

$$
n_{4}=c m_{2}+c^{\prime}\left(m_{2}+m_{1}\right)>\left(c+c^{\prime}\right) m_{2}>96 m_{2}>48 \frac{\log D}{\log 2},
$$

since $\sqrt{D}<2^{m_{2}}$. On applying Lemma 23 with (73), we obtain $D<2^{20}<10^{12}$. Thus $N(D)=3$. All cases are considered and the assertion is proved.

The combination of Assertions 1 and 2 yields the theorem.

\section{ACKNOWLEDGMENT}

The author would like to thank Professor A. Schinzel and the referee for their valuable suggestions. 


\section{REFERENCES}

1. F. Beukers, On the generalized Ramanujan-Nagell equation. I, Acta Arith. 38 (1980/1981), 389-410.

2. L.-K. Hua, Introduction to number theory, Springer-Verlag, Berlin, 1982.

3. R. Lidl and H. Niederreiter, Finite fields, Addison-Wesley, Reading, Mass., 1983.

4. T. Nagell, Contributions to the theory of a category of diophantine equations of the second degree with two unknowns, Nova Acta Soc. Sci. Upsala (4) 16 (1955), 38 pp.

Research Department, Changsha Railway Institute, Changsha, Hunan, Peoples RePUBLIC OF CHINA 